\section{OPEN ACCESS}

Edited by:

Yimin Li,

Guangzhou Medical University, China

Reviewed by:

Jianfeng $W u$,

The First Affiliated Hospital of

Sun Yat-Sen University, China

Johan Van Weyenbergh,

KU Leuven, Belgium

Shuwang $\mathrm{Ge}$

Huazhong University of Science and

Technology, China

*Correspondence:

Dechang Chen

chendechangsh@hotmail.com

Jean-Louis Tebou

jean-louis.teboul@aphp.fr

Diillali Annane

djillali.annane@aphp.fr

${ }^{\dagger}$ These authors have contributed equally to this work

Specialty section: This article was submitted to Viral Immunology,

a section of the journal

Frontiers in Immunology

Received: 28 February 2021 Accepted: 07 July 2021

Published: 02 August 2021

Citation:

Liu J, Shen Y, Wen Z, Xu Q, Wu Z, Feng H, Li Z, Dong X, Huang S, Guo J,

Zhang L, Chen Y, Li W, Zhu W, Du H,

Liu Y, Wang T, Chen L, Teboul J-L, Annane D and Chen D (2021)

Efficacy of Thymosin Alpha 1 in the Treatment of COVID-19: A

Multicenter Cohort Study.

Front. Immunol. 12:673693. doi: 10.3389/fimmu.2021.673693

\title{
Efficacy of Thymosin Alpha 1 in the Treatment of COVID-19: A Multicenter Cohort Study
}

\begin{abstract}
Jiao $\mathrm{Liu}^{1 \dagger}$, Yanfei Shen ${ }^{2 \dagger}$, Zhenliang Wen ${ }^{1 \dagger}$, Qianghong $\mathrm{Xu}^{2 \dagger}$, Zhixiong $\mathrm{Wu}^{3 \dagger}$, Huibin Feng ${ }^{4 t}$, Zhongyi $\mathrm{Li}^{5+}$, Xuan Dong ${ }^{6}$, Sisi Huang ${ }^{1}$, Jun Guo ${ }^{7}$, Lidi Zhang ${ }^{1}$, Yizhu Chen ${ }^{1}$, Wenzhe $\mathrm{Li}^{1}$, Wei Zhu ${ }^{8}$, Hangxiang Du ${ }^{1}$, Yongan Liu ${ }^{1}$, Tao Wang ${ }^{1}$, Limin Chen $^{1}$, Jean-Louis Teboul ${ }^{9 *}$, Djillali Annane ${ }^{10^{*}}$ and Dechang Chen ${ }^{1 *}$
\end{abstract}

\begin{abstract}
1 Department of Critical Care Medicine, Ruijiin Hospital, Shanghai Jiao Tong University School of Medicine, Shanghai, China, 2 Department of Critical Care Medicine, Zhejiang Hospital, Hangzhou, China, ${ }^{3}$ Department of Surgical Intensive Care Unit, Huadong Hospital Affiliated to Fudan University, Shanghai, China, ${ }^{4}$ Intensive Care Unit, Huangshi Central Hospital, Affiliated Hospital of Hubei Polytechnic University, Huangshi, China, ${ }^{5}$ Department of Critical Care Medicine, Wuhan No.9 Hospital, Wuhan, China, ${ }^{6}$ Tuberculosis and Respiratory Department, Wuhan Jinyintan Hospital, Wuhan, China, ${ }^{7}$ Intensive Care Unit, Huazhong University of Science and Technology Union Jiangbei Hospital, Wuhan, China, ${ }^{8}$ Intensive Care Unit, Tianyou Hospital Affiliated to Wuhan University of Science \& Technology, Wuhan, China, ${ }^{9}$ Service de Médecine-Intensive Réanimation, Hôpital Bicêtre, AP-HP. Université Paris-Saclay, Inserm UMR 999, Université Paris-Saclay, Le Kremlin-Bicêtre, France, ${ }^{10}$ Department of Intensive Care, Hôpital Raymond Poincaré (APHP), Laboratory of Infection \& Inflammation - U1173, School of Medicine Simone Veil, University Versailles Saint Quentin - University Paris Saclay, INSERM, Garches, France
\end{abstract}

Background: Thymosin alpha $1(T \alpha 1)$ is widely used to treat patients with COVID-19 in China; however, its efficacy remains unclear. This study aimed to explore the efficacy of To1 as a COVID-19 therapy.

Methods: We performed a multicenter cohort study in five tertiary hospitals in the Hubei province of China between December 2019 and March 2020. The patient non-recovery rate was used as the primary outcome.

Results: All crude outcomes, including non-recovery rate (65/306 vs. 290/1,976, $p=$ 0.003), in-hospital mortality rate (62/306 vs. 271/1,976, $p=0.003)$, intubation rate (31/ 306 vs. 106/1,976, $p=0.001$ ), acute respiratory distress syndrome (ARDS) incidence (104/306 vs. 499/1,976, $p=0.001)$, acute kidney injury (AKI) incidence (26/306 vs. 66/ $1,976, p<0.001)$, and length of intensive care unit (ICU) stay $(14.9 \pm 12.7 \mathrm{vs} .8 .7 \pm 8.2$ days, $p<0.001$ ), were significantly higher in the T $\alpha 1$ treatment group. After adjusting for

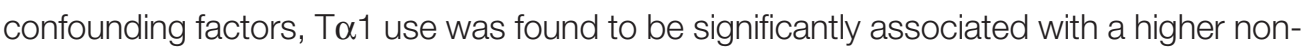
recovery rate than non-T $\alpha 1$ use (OR 1.5, 95\% $\mathrm{Cl} 1.1-2.1, p=0.028)$. An increased risk of non-recovery rate associated with $T \alpha 1$ use was observed in the patient subgroups with maximum sequential organ failure assessment (SOFA) scores $\geq 2$ (OR 2.0, 95\% Cl 1.4-2.9, $p=0.024$ ), a record of ICU admission (OR 5.4, 95\% Cl 2.1-14.0, $p<0.001$ ), and lower $\mathrm{PaO} 2 / \mathrm{FiO} 2$ values (OR 1.9, 95\% Cl 1.1-3.4, $p=0.046)$. Furthermore, later initiation of T $\alpha 1$ use was associated with a higher non-recovery rate.

Conclusion: T $\alpha 1$ use in COVID-19 patients was associated with an increased nonrecovery rate, especially in those with greater disease severity.

Keywords: thymosin alpha 1, COVID-19, non-recovery, disease severity, efficacy 


\section{INTRODUCTION}

Coronavirus 2019 disease (COVID-19) represents an ongoing global threat to human health and has caused more than 2,400,000 deaths to date (JHU data-23/02/2021, https:// coronavirus.jhu.edu/). As the COVID-19 outbreak continues, novel and effective therapies are urgently needed.

Current evidence indicates that immune dysfunction in COVID-19 plays a critical role in disease severity and is associated with a poor prognosis. Several studies have reported that inflammatory markers such as C-reactive protein (CRP), ferritin, interleukin (IL)-6, IL-10, and tumor necrosis factor- $\alpha$ $($ TNF- $\alpha$ ) were significantly elevated in severe COVID-19 cases. The resulting cytokine storm is thought to cause rapid COVID19 progression and increased deaths (1-3). Furthermore, Yu and colleagues (4) concluded that adaptive immune response dysregulation contributes to the cytokine storm and further leads to severe COVID-19. Dozens of studies have been performed to explore potential medical interventions to attenuate the harmful immune response, including statins (5), corticosteroids $(6,7)$, and thymosin alpha 1 (To1) (8-11). However, the benefits of immunomodulatory treatment of patients with COVID-19 have remained controversial.

$\mathrm{T} \alpha 1$ is an endogenous polypeptide hormone secreted by thymic epithelial cells (12). As an immunomodulatory therapy $(12,13), T \alpha 1$ has been investigated in many diseases involving immune dysfunction [such as sepsis, cystic fibrosis, and hepatitis viral infection (14-16)] and is associated with an improved patient outcome. The efficacy of T $\alpha 1$ in COVID-19 has also been investigated in several studies (8-11). However, the conclusions remain inconsistent. For instance, in a retrospective study including 76 severe COVID-19 cases (8), Liu et al. reported that $\mathrm{T} \alpha 1$ use could increase $\mathrm{T}$ cell numbers and was associated with reduced mortality. However, other studies found that $\mathrm{T} \alpha 1$ use showed no effect on mortality in COVID-19 (9) or was even associated with increased mortality in patients with COVID-19 of all severity levels (10). The reasons behind these inconsistent findings remain unclear. However, emerging evidence indicated that COVID-19 is a heterogeneous disease $(17,18)$. Whether the role of T $\alpha 1$ in COVID-19 is affected by different phenotypes remains unclear.

To this end, we performed a large multicenter cohort study to explore the efficacy of To1 in COVID-19 patients displaying a range of disease severities. Interaction analysis between $T \alpha 1$ and predefined parameters was also performed to identify the heterogeneous effectiveness of $\mathrm{T} \alpha 1$ in different patient subgroups.

\section{METHODS}

\section{Study Setting and Design}

This was a multicenter cohort study conducted at five tertiary hospitals (in the Hubei province of China) that were designated

Abbreviations: SOFA, sequential organ failure assessment; ICU, intensive care unit; $\mathrm{PaO} 2 / \mathrm{FiO} 2$, ratio of partial pressure of arterial oxygen to fraction of inspired oxygen; ARDS, acute respiratory distress syndrome. by the Chinese government as COVID-19 treatment sites: including Union Jiangbei Hospital, Wuhan No. 9 Hospital, Wuhan No. 4 Hospital, Wuhan Jinyintan Hospital, and Huangshi Central Hospital. This study was approved by the Ethics Committee of the Jin Yin-tan Hospital (KY-2020-03.01), and patient informed consent was waived due to the retrospective nature of this study. All the patients admitted to the five tertiary hospitals between December 29, 2019 and March 16,2020 were screened and included in the study if the following inclusion criteria were met: 1 . Confirmed COVID-19 diagnosis made according to the Chinese COVID-19 diagnosis and treatment guidelines (Trial Version 7) (19); and 2. Age over 18 years. Patients were excluded if their hospital record was incomplete. No other exclusion criteria were applied.

\section{Data Extraction}

Demographic characteristics, smoking and comorbidities data, and initial laboratory indices recorded within the first $24 \mathrm{~h}$ after hospital admission were extracted from each center through electronic medical records. The change in lymphocyte counts was defined as the difference between the initial lymphocyte count and the minimum lymphocyte count. Clinical classification, special drug combinations, and clinical outcomes data were also recorded. The sequential organ failure assessment (SOFA) scores (20) and acute physiology and chronic health evaluation II (APACHE II) scores (21) were calculated to assess the severity of illness using data obtained in the first $24 \mathrm{~h}$ after hospital admission. T $\alpha 1$ use was defined as any record of subcutaneous/intramuscular T $\alpha 1$ administration throughout the entire hospital stay. Both the duration and timing of To1 use were also extracted.

\section{COVID-19 Severity Classification}

The detailed diagnosis of COVID-19 (19) was established as follows: 1. patients with an epidemiological history and chest imaging (computed tomography or radiography) that suggested viral pneumonia; 2. laboratory-confirmed SARS-COV-2 infection of throat swab, sputum, and/or lower respiratory tract samples by high-throughput sequencing or real-time reverse transcriptase polymerase chain reaction (RT-PCR); or 3. confirmed plasma positivity for specific antibody (IgM or/and IgG) against SARS-COV-2.

COVID-19 disease severity was classified according to the Chinese diagnosis and COVID-19 treatment guidelines (Trial Version 7) (19). Briefly, patients were diagnosed with moderately severe COVID-19 if they had fever, respiratory symptoms, and chest imaging (computed tomography or radiography) suggesting viral pneumonia. Patients who met either conditions i-iii or iv-vi of the following criteria were diagnosed as having severe or critical disease, respectively: (i) respiratory rate $\geq 30$ beats/min; (ii) resting stable oxygen saturation (SpO2) $\leq 93 \%$; (iii) $\mathrm{PaO} 2 / \mathrm{FiO} 2 \leq 300 \mathrm{mmHg}$ ( 1 $\mathrm{mmHg}=0.133 \mathrm{kPa}$ ); (iv) respiratory failure requiring mechanical ventilation; (v) shock; or (vi) multiple organ failure requiring ICU admission.

Diagnosis of acute respiratory distress syndrome (ARDS) was made according to the Berlin definition (22). Acute kidney injury 
(AKI) was defined according to the "Kidney Disease: Improving Global Outcomes" document (23).

\section{Stratification and Outcome Definition}

Subgroup analysis was performed to interpret possible interactions between T $\alpha 1$ use and disease severity. Data were stratified according to the median of the maximum SOFA score $(\geq /<2), \mathrm{PaO} 2 / \mathrm{FiO} 2$ at admission, admission to intensive care unit (ICU), application of mechanical ventilation, and development of ARDS. Non-recovery rate was the primary outcome at the time of data collection. Non-recovering patients were those who died as a result of COVID-19 or who were still in hospital, but in any one of severely deteriorated condition: a. after sufficient fluid resuscitation $(\geq 30 \mathrm{ml} / \mathrm{kg}$ crystalloid solution), a dose of vasopressor therapy equal to or greater than an equivalent of norepinephrine $0.4 \mu \mathrm{g} / \mathrm{kg} / \mathrm{min}$ for at least $24 \mathrm{~h}$ was hard to maintain mean arterial pressure (MAP) at $65 \mathrm{mmHg}$; b. $\mathrm{PaO} 2 / \mathrm{FiO} 2$ was continuously lower than 100 $\mathrm{mmHg}$ for $24 \mathrm{~h}$ even with invasive mechanical ventilation. Secondary outcomes included in-hospital mortality, ICU admission, intubation rate, ARDS incidence, AKI incidence, duration of mechanical ventilation, length of ICU stay, and length of hospital stay. Sensitivity analysis was also performed using in-hospital mortality as the primary outcome.

\section{Management of Missing Data}

Most continuous variables in the study had less than 5\% missing data and were therefore replaced with their mean or median values. Variables with more than $20 \%$ missing data were not completed. For dichotomous variables, the missing value was replaced with the default value (zero).

\section{Statistical Analysis}

Continuous variables were presented as mean \pm standard deviation (SD) or median (interquartile range, IQR) according to the data distribution. The Student's t-test or Wilcoxon ranksum test were used as appropriate. Categorical data were compared using the Chi-squared test and were presented as percentages. To adjust for potential confounding factors, variables with $p<0.2$ in the univariate comparison were included in the initial model. Stepwise regression was used to build the final logistic model. Multicollinearity was tested using the variance inflation factor (VIF) method, with a VIF $\geq 5$ suggesting potential multicollinearity. To test the stability of these logistic models, bootstrap analysis (using 100 resamples) was performed.

The significant impact of maximum SOFA scores on the association between $\mathrm{T} \alpha 1$ use and the non-recovery rate was detected in the multivariable analysis. Thus, interactions between $\mathrm{T} \alpha 1$ use and disease severity indices were explored, and subgroup analysis was performed according to the following five parameters: median SOFA score $(\geq /<2)$, ICU admission, $\mathrm{PaO} 2 / \mathrm{FiO} 2$ value, application of mechanical ventilation, and development of ARDS. For interpretation, the predicted marginal effect of T $\alpha 1$ was estimated at different SOFA scores and $\mathrm{PaO} 2 / \mathrm{FiO} 2$ values.
Propensity score matching (PSM) was applied to minimize the impact of confounding factors, such as biochemical indices and the disease severity score, which may lead to a biased outcome. The propensity score was assigned based on the probability that a patient would receive $\mathrm{T} \alpha 1$ therapy and estimated using a multivariable logistic regression model. A one-to-three nearest neighbor matching algorithm was applied with a caliper of 0.02 . The following variables were selected to generate the propensity score: age, hypertension, chronic obstructive pulmonary disease (COPD), chronic liver disease, chronic cardiac disease, maximum SOFA score, ICU admission, serum hemoglobin and creatinine levels, and initial white blood cell, lymphocyte, and platelet counts. The bias in the means (or proportions) of covariates between two groups was examined using the standardized difference before and after PSM. Finally, to test bias owing to imbalances in unmeasured covariates in the PSM, sensitivity analyses were performed to quantify the degree of hidden bias (Gamma value) that would need to be present to invalidate our main conclusions. A two-tailed test was performed, and $p<0.05$ was considered statistically significant. All statistical analyses were performed using STATA 14.0 (College Station, TX, USA).

\section{RESULTS}

\section{Baseline Characteristics and Crude Comparisons}

Of the 2,411 patients admitted to the five participating tertiary hospitals, 73 were excluded because of unconfirmed COVID-19, 51 were excluded because of duplicate records, and 5 were excluded due to missing outcome data. Of the remaining 2,282 patients that were eligible to participate in our study, 306 received T $\alpha 1$ therapy (Figure 1). Clinical characteristics associated with COVID-19 patients in the T $\alpha 1$ and non-T $\alpha 1$ treatment groups are shown in Table 1. There were no significant differences in terms of age or gender between the two groups. More patients received corticosteroids (107/306 vs. $382 / 1,976, p<0.001)$ and interferon $(74 / 306$ vs. 108/1,976, $p<0.001)$ therapy in the T $\alpha 1$ group than in the non-T $\alpha 1$ group. Patients who received T $\alpha 1$ therapy had almost the same disease severity according to the SOFA score, APACHE II score, initial serum creatinine and maximum lactate levels, although the patients in the T $\alpha 1$ group included a slightly larger proportion of critically ill patients (59/306 vs. 240/1,976). Furthermore, although both the initial and minimal lymphocyte counts were significantly lower in the T $\alpha 1$ group than in the non-T $\alpha 1$ group $\left(1.0 \pm 0.5 v s .1 .1 \pm 0.6 \times 10^{\wedge} 9 / \mathrm{L}, p=\right.$ $0.007 ; 0.8 \pm 0.5$ vs. $0.9 \pm 0.6 \times 10^{\wedge} 9 / \mathrm{L}, p<0.001$ for the T $\alpha 1$ and non-T $\alpha 1$ groups, respectively), the change in lymphocyte count was comparable between the groups $(0.1 \pm 0.3$ vs. $0.1 \pm 0.3 \times$ $\left.10^{\wedge} 9 / \mathrm{L}, p=0.337\right)$. However, as reported in Table 2, all crude outcomes, including non-recovery rate (65/306 vs. 290/1,976, $p=$ $0.003)$, in-hospital mortality rate (62/306 vs. 271/1,976, $p=$ $0.003)$, intubation rate $(31 / 306$ vs. $106 / 1,976, p=0.001)$, ARDS incidence $(104 / 306$ vs. $499 / 1,976, p=0.001)$, AKI incidence $(26 /$ 


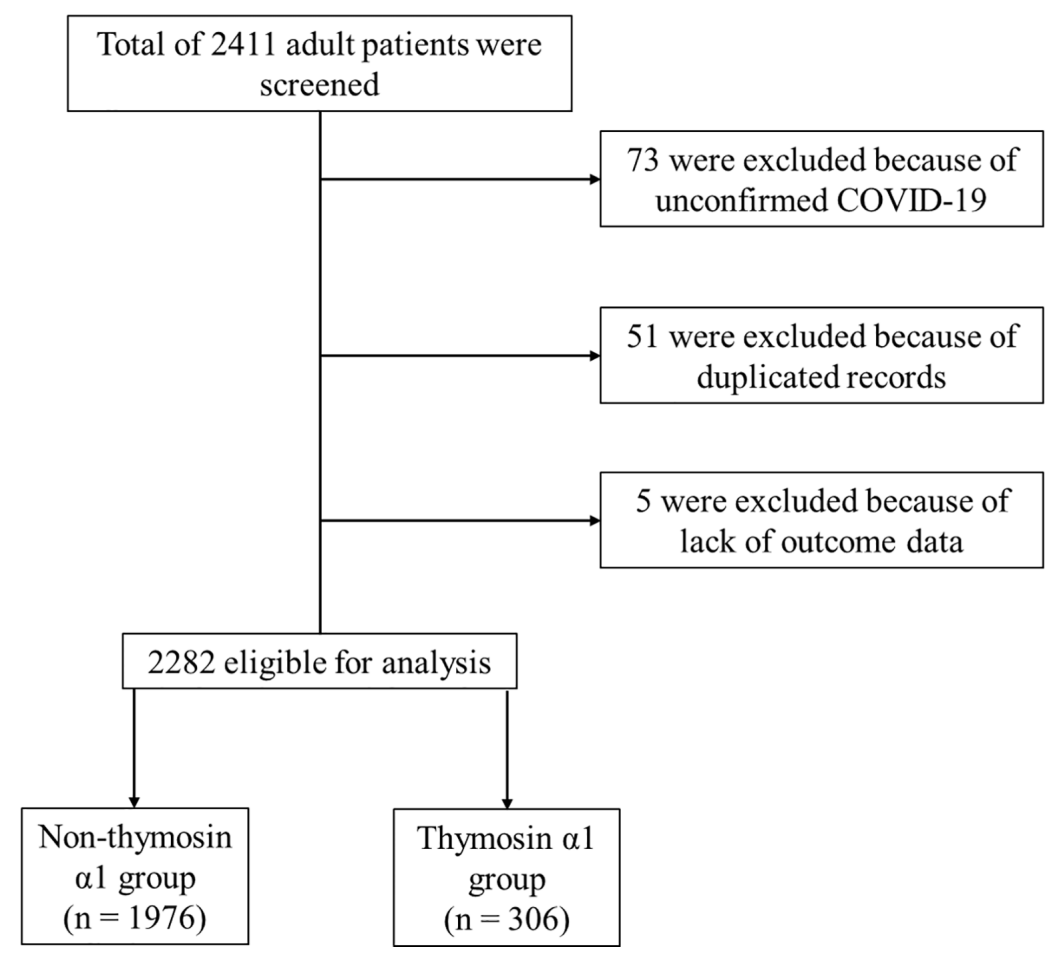

FIGURE 1 | Flow chart of the present study.

306 vs. 66/1,976, $p<0.001)$, and Length of ICU stay $(14.9 \pm 12.7$ vs. $8.7 \pm 8.2$ days, $p<0.001$ ), were significantly higher in the T $\alpha 1$ group than in the non-T $\alpha 1$ group. Information relating to the number of patients, T $\alpha 1$ use, and the non-recovery rate for each center is provided in Supplementary Table S1.

\section{Adjusted Association Between Ta1 Use and Non-Recovery Rate}

Potential confounding factors were adjusted for in the multivariable logistic models as shown in the Table 3. The odds ratio $(\mathrm{OR})$ for the non-recovery rate of $\mathrm{T} \alpha 1$ use was found to be significant (Model 2: OR 1.5, 95\% confidence interval (CI) 1.1-2.1, $p=0.028$ ) using bootstrap analysis with 1,000 resamples. However, after the addition of the maximum SOFA score to Model 3, the association between T $\alpha 1$ use and the non-recovery rate became non-significant (OR 1.4, 95\% CI 0.92.2, $p=0.118$ ).

Interactions between $\mathrm{T} \alpha 1$ use and different disease severity indices were evaluated. There were significant interactions between T $\alpha 1$ use and the maximum SOFA score $(p=0.024)$ and ICU admission ( $p<0.001$ ) (Figure 2). T $\alpha 1$ use was significantly associated with an increase in non-recovery rate in the subgroup with a maximum SOFA score $\geq 2$ (OR 2.0, 95\% CI 1.4-2.9) or ICU admission (OR 5.4, 95\% CI 2.1-14.0), whereas this association was not significant in subgroups with a SOFA score $<2$ (OR 0.6, 95\% CI 0.24-1.7) or without ICU admission (OR 1.1, 95\% CI 0.7-1.7). There was no significant interaction between $\mathrm{T} \alpha 1$ use and $\mathrm{PaO} 2 / \mathrm{FiO} 2$ values $(\geq 300$
mmHg) at admission, mechanical ventilation, or ARDS development. However, a trend towards higher ORs was observed in subgroups with low $\mathrm{PaO} 2 / \mathrm{FiO} 2$ values $(<300$ $\mathrm{mmHg}$ ), mechanical ventilation, and ARDS. Furthermore, significant interactions were also observed using maximum SOFA $(p=0.003)$ and $\mathrm{PaO} 2 / \mathrm{FiO} 2$ values at admission $(p=$ $0.016)$ as continuous variables. The predicted marginal effect of $\mathrm{T} \alpha 1$ on the non-recovery rate was estimated using different SOFA and $\mathrm{PaO} 2 / \mathrm{FiO} 2$ values (Supplementary Figure S1). The difference in the probability of non-recovery between the T $\alpha 1$ and non-T $\alpha 1$ groups correlated positively with the SOFA score or negatively with the $\mathrm{PaO} 2 / \mathrm{FiO} 2$ value. In addition, aiming to evaluate whether the effect of $\mathrm{T} \alpha 1$ administration was influenced by other concurrent immunotherapies, we tested the potential interaction between T $\alpha 1$, corticosteroids, and interferon and found no significant interaction. We also included both the corticosteroids and interferon factors in our multivariable model, and the overall results in each subgroup remained unchanged (Supplementary Table S2). The proportions of patients receiving T $\alpha 1$, corticosteroids, and/or interferon are presented in a Venn diagram (Supplementary Figure S2).

PSM was performed using a 1:3 algorithm; 306 cases from the T $\alpha 1$ group and 720 cases from the non-T $\alpha 1$ group were well matched (Supplementary Tables S3, 4). The overall quality of the matched sample was assessed by comparing the standardized difference of included covariates before and after PSM. There was no significant difference between the matched groups relating to 
TABLE 1 | Baseline comparisons between thymosin $\alpha 1$ and non-thymosin $\alpha 1$ groups.

\begin{tabular}{|c|c|c|c|}
\hline Variables & Non-thymosin $\alpha 1$ group $(n=1,976)$ & Thymosin $\alpha 1$ group $(n=306)$ & $p$ \\
\hline Age (years) & $58.4 \pm 14.5$ & $57.9 \pm 14.5$ & 0.601 \\
\hline \multicolumn{4}{|l|}{ Gender, n (\%) } \\
\hline Male & 1025 (51.9) & $165(53.9)$ & \multirow[t]{2}{*}{0.504} \\
\hline Female & $951(48.1)$ & $141(46.1)$ & \\
\hline Smoking, n (\%) & $54(2.7)$ & $7(2.2)$ & $<0.001$ \\
\hline \multicolumn{4}{|l|}{ Comorbidities, n (\%) } \\
\hline Hypertension & $525(26.5)$ & $98(32.0)$ & 0.046 \\
\hline Diabetes mellitus & $247(12.5)$ & $36(11.7)$ & 0.717 \\
\hline Chronic heart diseases & $182(9.2)$ & $18(5.8)$ & 0.055 \\
\hline Chronic obstructive pulmonary disease & $39(1.9)$ & $9(2.9)$ & 0.272 \\
\hline Chronic renal diseases & $54(2.7)$ & $6(1.9)$ & 0.432 \\
\hline Malignant tumor & $50(2.5)$ & $7(2.2)$ & 0.800 \\
\hline \multicolumn{4}{|l|}{ Disease severity scores [median (IQR)] } \\
\hline SOFA score on admission & $1(0-2)$ & $1(0-2)$ & 0.915 \\
\hline Maximum SOFA score & $1(0-2)$ & $1(0-3)$ & 0.926 \\
\hline APACHE II on admission & $5(3-7)$ & $5(3-7)$ & 0.949 \\
\hline Maximum APACHE II score & $5(3-7)$ & $5(3-8)$ & $<0.001$ \\
\hline Clinical classification, $\mathbf{n}(\%)$ & & & $<0.001$ \\
\hline Moderate type & $1276(64.6)$ & $186(60.8)$ & \\
\hline Severe type & $460(23.3)$ & $61(19.9)$ & \\
\hline Critical type & $240(12.1)$ & 59 (19.3) & \\
\hline \multicolumn{4}{|l|}{ Laboratory finding } \\
\hline $\mathrm{PaO} 2 / \mathrm{FiO} 2$ & $279.3 \pm 144.2$ & $316.1 \pm 107.7$ & 0.002 \\
\hline Initial white blood cell count (10^9/L) & $6.9 \pm 3.6$ & $6.9 \pm 4.0$ & 0.977 \\
\hline Initial lymphocyte cell count (10^9/L) & $1.1 \pm 0.6$ & $1.0 \pm 0.5$ & 0.007 \\
\hline Minimum lymphocyte cell count (10^9/L) & $0.9 \pm 0.6$ & $0.8 \pm 0.5$ & $<0.001$ \\
\hline Change of lymphocyte cell count (10^9/L) & $0.1 \pm 0.3$ & $0.1 \pm 0.3$ & 0.337 \\
\hline Initial hemoglobin level (g/dl) & $122.7 \pm 17.0$ & $123.4 \pm 18.2$ & 0.462 \\
\hline Initial platelet count (10^9/L) & $217.4 \pm 89.5$ & $207.5 \pm 87.0$ & 0.071 \\
\hline Initial albumin level (g/l) & $34.3 \pm 5.0$ & $32.4 \pm 4.8$ & $<0.001$ \\
\hline Initial serum creatinine (mmol/l) & $75.9 \pm 51.9$ & $82.1 \pm 66.6$ & 0.063 \\
\hline Initial serum sodium (mmol/L) & $139.7 \pm 4.2(n=1,606)$ & $140.8 \pm 3.2(n=261)$ & $<0.001$ \\
\hline Maximum lactate level (mmol/L) & $2.5 \pm 2.4(n=366)$ & $2.4 \pm 2.0(n=10)$ & 0.913 \\
\hline \multicolumn{4}{|l|}{ Medicine, n (\%) } \\
\hline Corticosteroids & 382 (19.3) & $107(35.0)$ & $<0.001$ \\
\hline Interferon & $158(8.0)$ & $74(24.2)$ & $<0.001$ \\
\hline Ribavirin & $95(4.8)$ & $4(1.3)$ & 0.005 \\
\hline Oseltamivir & $46(2.3)$ & $6(2.0)$ & 0.689 \\
\hline
\end{tabular}

PaO2/FiO2, ratio of partial pressure of arterial oxygen to fraction of inspired oxygen; SOFA, sequential organ failure assessment; APACHE II, acute physiology and chronic health evaluation II.

TABLE 2 | Clinical outcomes of overall COVID-19 patients with or without thymosin $\alpha 1$ treatment.

\begin{tabular}{|c|c|c|c|}
\hline Clinical outcomes & Non-thymosin $\alpha 1$ group $(n=1,976)$ & Thymosin $\alpha 1$ group $(n=306)$ & $p$ \\
\hline Non-recovery, n (\%) & $290(14.6)$ & $65(21.2)$ & 0.003 \\
\hline In-hospital mortality, n (\%) & $271(13.7)$ & $62(20.2)$ & 0.003 \\
\hline ICU admission, $\mathrm{n}(\%)$ & $287(14.5)$ & $42(13.7)$ & 0.711 \\
\hline Intubation, $\mathrm{n}(\%)$ & $106(5.3)$ & $31(10.1)$ & 0.001 \\
\hline Duration of mechanical ventilation (days) & $6.2 \pm 4.8$ & $6.7 \pm 4.2$ & 0.644 \\
\hline Length of ICU stay (days) & $8.7 \pm 8.2$ & $14.9 \pm 12.7$ & $<0.001$ \\
\hline Length of hospital stay (days) & $13.8 \pm 7.9$ & $12.1 \pm 8.2$ & $<0.001$ \\
\hline
\end{tabular}

ICU, intensive care unit.

all 11 covariates (Supplementary Table S3). However, all clinical outcomes, including the non-recovery rate (65/306 vs. 111/720, $p=0.024)$, intubation rate $(31 / 306 v s .43 / 720, p=0.018)$, ARDS incidence $(104 / 306$ vs. 196/720, $p=0.029)$, and AKI incidence
(26/306 vs. 26/720, $p=0.001)$ were significantly higher in the T $\alpha 1$ group than in the non-T $\alpha 1$ group (Table 4). Furthermore, another PSM was performed in the subgroup with a maximum SOFA $\geq 2$, as the impact of T $\alpha 1$ use on the non-recovery rate was 
TABLE 3 | Associations between non-recovery rate and thymosin $\alpha 1$ use in different logistic models.

\begin{tabular}{|c|c|c|c|c|c|c|}
\hline \multirow[t]{2}{*}{ Variables } & \multicolumn{2}{|l|}{ Model 1} & \multicolumn{2}{|l|}{ Model 2} & \multicolumn{2}{|l|}{ Model 3} \\
\hline & Crude OR (95\% Cl) & $p$ & $\begin{array}{c}\text { Multivariable logistic } \\
\text { model with bootstrapping, } \\
\text { aORs }(95 \% \mathrm{Cl})\end{array}$ & $p$ & Adjusted aORs $(95 \% \mathrm{Cl})$ & $p$ \\
\hline Thymosin $\alpha 1$ use & $1.5(1.1-2.1)$ & 0.003 & $1.5(1.1-2.1)$ & 0.028 & $1.4(0.9-2.2)$ & 0.118 \\
\hline Age $>65$ & & & $3.4(2.6-4.4)$ & $<0.001$ & $3.9(2.8-5.3)$ & $<0.001$ \\
\hline Diabetes mellitus & & & $1.6(1.1-2.2)$ & 0.007 & $1.3(0.8-2.0)$ & 0.204 \\
\hline $\mathrm{PaO} 2 / \mathrm{FiO} 2<300$ & & & $2.3(1.7-3.1)$ & $<0.001$ & $0.6(0.4-0.9)$ & 0.042 \\
\hline Lymphocyte counts & & & $0.4(0.3-0.5)$ & $<0.001$ & $0.5(0.4-0.7)$ & $<0.001$ \\
\hline Platelet counts & & & $0.9(0.9-0.9)$ & $<0.001$ & $0.9(0.9-0.9)$ & 0.287 \\
\hline Creatinine level & & & $1.0(1.0-1.0)$ & 0.001 & $0.9(0.9-1.0)$ & 0.108 \\
\hline Union Jiangbei Hospital & & & Ref. & - & Ref. & - \\
\hline Wuhan No.9 Hospital & & & $1.0(0.6-1.6)$ & 0.979 & $0.1(0.06-0.2)$ & $<0.001$ \\
\hline Wuhan No.4 Hospital & & & $3.0(1.5-6.0)$ & 0.002 & $0.5(0.2-1.0)$ & 0.062 \\
\hline Wuhan Jinyintan Hospital & & & $1.4(0.9-2.3)$ & 0.117 & $0.1(0.1-0.3)$ & $<0.001$ \\
\hline Huangshi Central Hospital & & & $6.0(2.6-14.2)$ & $<0.001$ & $0.4(0.2-1.2)$ & 0.114 \\
\hline Maximum SOFA score & & & & & $1.9(1.8-2.1)$ & $<0.001$ \\
\hline
\end{tabular}

Three logistic models were used to evaluate the association between non-recovery rate and thymosin $\alpha 1$ use. OR of thymosin $\alpha 1$ use was significantly associated with increased nonrecovery rate in models 1 and 2. However, after adjusting for SOFA score in model 3, the OR of thymosin $\alpha 1$ became non-significant. Bootstrap techniques (100 resamples) was used for calculating $95 \% \mathrm{Cl}$ in models 2 and 3.

OR, odds ratio; aORs, adjusted odds ratios; PaO2/FiO2, ratio of partial pressure of arterial oxygen to fraction of inspired oxygen; SOFA, sequential organ failure assessment.

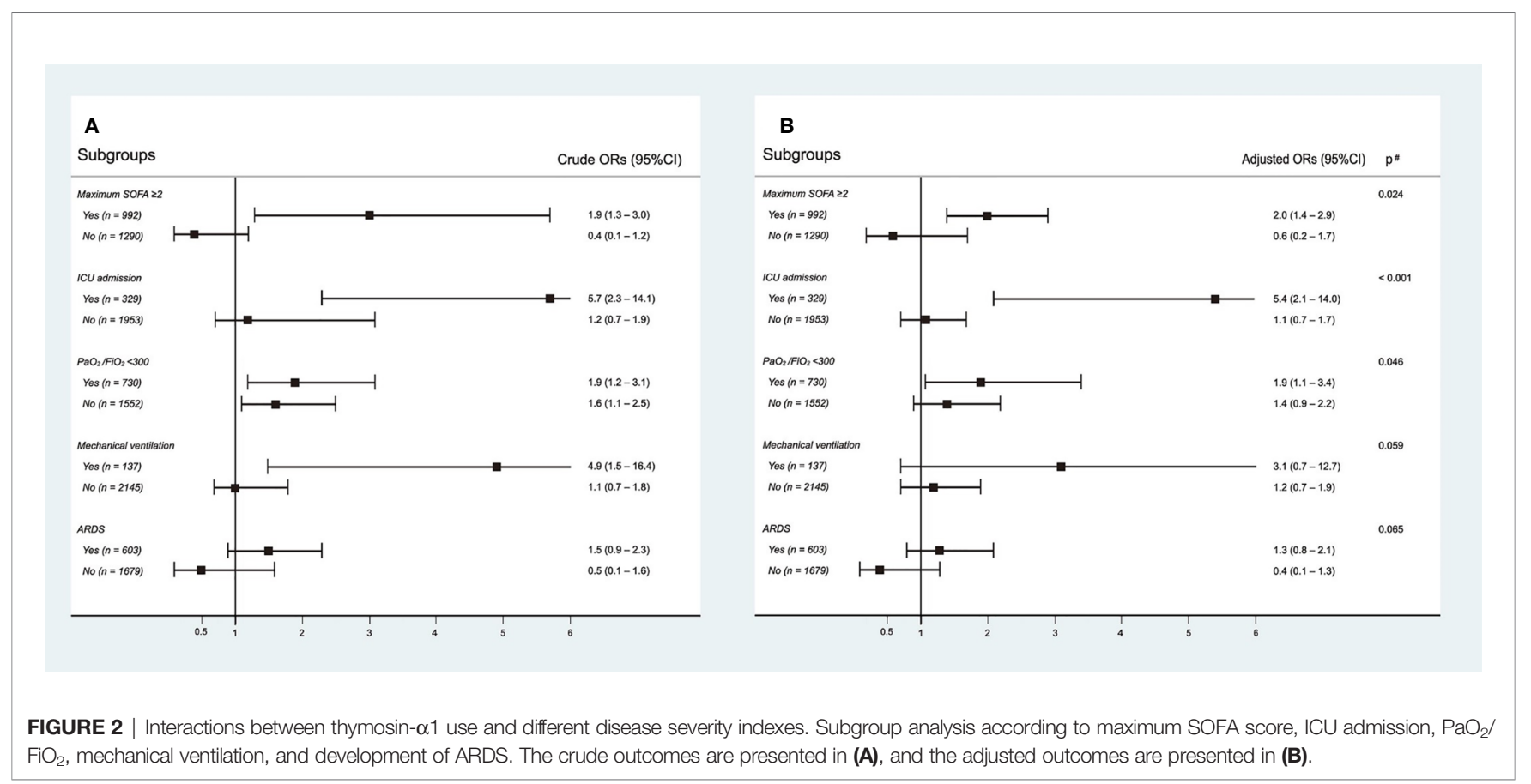

significantly affected by disease severity, and all the above conclusions were supported (Table 4).

\section{Sensitivity Analysis}

In the non-recovery group $(n=355)$, there are 18 patients still under treatment, but in a severely deteriorated condition during data extraction. For robustness, we performed sensitivity analysis under three patterns, and "death" was used as the dependent outcome (Supplementary Table S4). In pattern 1, these 18 patients were divided into the "alive" group. In pattern 2, these 18 patients were divided into the "death" group. In pattern 3, these 18 patients were excluded from the analysis. The results were stable in all these three patterns. In addition, interactions between thymosin $\alpha 1$ use and disease severity indexes on in-hospital mortality are also presented in Supplementary Table S5.

\section{Association Between Duration/Timing of To1 Treatment and Patient Non-recovery Rate}

Both the duration and timing of $T \alpha 1$ were included in the multivariable logistic model in different forms (Table 5, Model 1: continuous variable; Model 2: dichotomous variable according to 
TABLE 4 | Comparisons of clinical outcomes after propensity score matching.

\begin{tabular}{|c|c|c|c|c|c|c|}
\hline \multirow{2}{*}{$\begin{array}{l}\text { Clinical } \\
\text { outcomes }\end{array}$} & \multicolumn{3}{|c|}{ All patients } & \multicolumn{3}{|c|}{ Patients with SOFA $\geq 2$} \\
\hline & $\begin{array}{l}\text { Non-thymosin } \alpha 1 \text { group } \\
\qquad(\mathrm{n}=720)\end{array}$ & $\begin{array}{l}\text { Thymosin } \alpha 1 \text { group } \\
\text { (n = 306) }\end{array}$ & $p$ & $\begin{array}{l}\text { Non-thymosin } \alpha 1 \text { group } \\
\qquad(\mathrm{n}=302)\end{array}$ & $\begin{array}{l}\text { Thymosin } \alpha 1 \text { group } \\
\text { ( } n=142)\end{array}$ & $p$ \\
\hline Non-recovery, n (\%) & $111(15.4)$ & $65(21.2)$ & 0.024 & $90(29.8)$ & $59(41.5)$ & 0.014 \\
\hline Intubation, n (\%) & $43(5.9)$ & $31(10.1)$ & 0.018 & $30(9.9)$ & $26(18.3)$ & 0.013 \\
\hline ARDS, n (\%) & $196(27.2)$ & $104(33.9)$ & 0.029 & $151(50.0)$ & $97(68.3)$ & $<0.001$ \\
\hline AKI, n (\%) & $26(3.6)$ & $26(8.5)$ & 0.001 & $22(7.2)$ & $23(16.2)$ & 0.004 \\
\hline
\end{tabular}

TABLE 5 | Association between the duration/timing of thymosin $\alpha 1$ use and non-recovery rate.

\begin{tabular}{|c|c|c|c|c|c|c|c|}
\hline Duration of thymosin $\alpha 1$ use (days) & ORs & $95 \% \mathrm{Cl}$ & $p$ & Timing of thymosin $\alpha 1$ use (days) & ORs & $95 \% \mathrm{Cl}$ & $p$ \\
\hline \multicolumn{4}{|l|}{ Model 1} & \multicolumn{4}{|l|}{ Model 1} \\
\hline \multicolumn{8}{|l|}{$(n=306)$} \\
\hline \multicolumn{4}{|l|}{ Model 2 (median value) } & \multicolumn{4}{|l|}{ Model 2 (median value) } \\
\hline$\leq 5(n=172)$ & Ref. & & & $\leq 3(n=159)$ & Ref. & & \\
\hline$>5(n=134)$ & 1.3 & $0.7-2.1$ & 0.404 & $>3(n=147)$ & 2.4 & $1.3-4.6$ & 0.007 \\
\hline \multicolumn{4}{|l|}{ Model 3 (tertile analysis) ( $p$ for trend: 0.574) } & \multicolumn{4}{|c|}{ Model 3 (tertile analysis) ( $p$ for trend: 0.005) } \\
\hline$\leq 3(n=106)$ & Ref. & & & $\leq 1(n=109)$ & Ref. & & \\
\hline $3-7(n=103)$ & 0.4 & $0.2-0.9$ & 0.037 & $2-10(n=91)$ & 4.1 & $1.5-10.7$ & 0.004 \\
\hline$\geq 7(n=97)$ & 1.2 & $0.6-2.5$ & 0.648 & $\geq 10(n=106)$ & 6.2 & $2.5-15.3$ & $<0.001$ \\
\hline
\end{tabular}

All models were adjusted for age, diabetes, PaO2/FiO2, lymphocyte and platelet count, serum creatinine level.

ORs, odds ratios.

median value; and Model 3: tertile analysis). To1 use at a later stage was significantly associated with a higher non-recovery rate than T $\alpha 1$ use at an earlier stage (Models 1-3). However, the duration of $\mathrm{T} \alpha 1$ use had a less significant effect on recovery rate. Inclusion of the maximum SOFA score as a confounding factor in the logistic model did not alter the results (Supplementary Table S6).

\section{DISCUSSION}

The current study has three major findings. First, T $\alpha 1$ use in COVID-19 was associated with poor clinical outcomes. This finding was confirmed both in the multivariable logistic model and via PSM. Second, there were significant interactions between T $\alpha 1$ use and disease severity indices, and the association between T $\alpha 1$ use and the non-recovery rate was stronger in severe COVID-19 cases. Third, T $\alpha 1$ use at a later stage was significantly associated with a higher non-recovery rate than $\mathrm{T} \alpha 1$ use at an earlier stage. However, the duration of T $\alpha 1$ use had a less significant effect on recovery rate.

Lymphopenia has been commonly reported in patients with severe COVID-19 $(4,24,25)$ and is associated with a poor outcome (24). Yu et al. (4) reported that a significant reduction in effector $\mathrm{T}$ cell numbers, accompanied by an increase in the frequency of effector B cells, was observed in COVID-19 patients with severe disease. This indicates that a decline in $\mathrm{T}$ cell numbers is likely the main cause of lymphopenia and may aggravate COVID-19 severity. Given the essential role of T cells in viral eradication, exploring medical interventions designed to boost $\mathrm{T}$ lymphocyte number and function may improve COVID19 prognosis (26). Previous studies have indicated that T $\alpha 1$ can promote $\mathrm{T}$ cell development and proliferation, increase their number, and enhance their function $(27,28)$. Yu's research $(4)$ also demonstrated that T $\alpha 1$ promoted the proliferation of effector $\mathrm{T}$ cells in vitro and relieved lymphopenia in COVID19 patients. Matteucci et al. (29) found that genes associated with cytokine signaling and expression were upregulated in patients with COVID-19, and the ex vivo treatment with T $\alpha 1$-mitigated cytokine expression, and inhibited lymphocyte activation. These findings provide the basis for the rationale use of T $\alpha 1$ in COVID-19, but these findings need to be further supported by clinical studies. A recent cohort study showed that T $\alpha 1$ can reduce mortality in patients with severe COVID-19 by reversing lymphocytopenia and restoring the function of exhausted T cells (8). However, bias risk should be considered when interpreting this conclusion, due to the limited sample size used (only 76 patients with severe COVID19 were included) and the presence of potential confounding factors. In another multicenter observation study, Wu et al. (11) also found that T $\alpha 1$ significantly decreased 28-day mortality and attenuated acute lung injury in critical type COVID-19 patients. However, the definition of critical type is largely different from our study. In addition, the disease severity was significantly imbalanced between $T \alpha 1$ and non-T $\alpha 1$ groups, as patients in the T $\alpha 1$ group had worse condition. Noteworthy, inconsistent findings were also 
reported in other studies. Sun et al. (9) reported that in 771 patients with COVID-19, use of T $\alpha 1$ was not associated with decreased mortality in critically ill COVID-19 patients. Wang et al. conducted a retrospective propensity score matched study including 317 COVID-19 patients, treatments with immunomodulatory therapies, including glucocorticoids, immunoglobulin, and thymosin, were significantly associated with a higher rate of COVID-19 death (10).

In the current study, T $\alpha 1$ was used in $13.4 \%$ of COVID-19 patients. We noted that $\mathrm{T} \alpha 1$ use was associated with poor prognoses, such as higher non-recovery and in-hospital mortality rate and higher ARDS and AKI incidence. After adjusting for potential confounders, T $\alpha 1$ use was still significantly associated with an increased non-recovery rate. However, the underlying mechanism remains unclear. One study (30) reported that T $\alpha 1$ exhibited a dual effect on dendritic cells (DCs) upon different pathogen encounter in vitro. T $\alpha 1$ was shown to promote DCs to secrete inflammatory cytokines, such as TNF- $\alpha$, IL-6, and IL-8, in response to viral infection. In contrast, T $\alpha 1$ dampened inflammation when the DCs were exposed to bacterial pathogens. Furthermore, as a result of the interaction analysis, we found that $\mathrm{T} \alpha 1$ use was associated with increased non-recovery rate mainly in patient subgroups with greater disease severity. Recent studies have found that COVID-19 follows a heterogeneous course of disease $(17,18)$. Shi et al. (31) categorized COVID-19 cases into three stages based on inflammatory status: stage I, an asymptomatic incubation period; stage II, non-severe symptomatic period; and stage III, severe respiratory symptomatic stage. For patients in stage III, a hyper-inflammatory response characterized by the overexpression of inflammatory factors and cytokines played a key role in patient deterioration (32). During this third stage, immunosuppressive therapy as opposed to immune enhancement therapy is needed (31). However, studies have indicated that $\mathrm{T} \alpha 1$ plays an important role in the activation and maturation of DCs, which in turn increases the secretion of inflammatory factors, promotes the differentiation of $\mathrm{T}$ cell precursors into Th1, eventually exacerbating the inflammatory response to viral infection (28, 33). In our study, the greatest disease severity indices, such as higher SOFA scores, lower $\mathrm{PaO} 2 / \mathrm{FiO} 2$ values, or admission to ICU, to a certain degree represented a more severe inflammatory stage. This may be the potential mechanism explaining why adjuvant T $\alpha 1$ use was associated with poor outcomes especially in severe COVID19 cases.

In addition, we found that the timing of T $\alpha 1$ treatment is also crucial as applying $\mathrm{T} \alpha 1$ at a later stage was significantly associated with increased non-recovery rate. Aiming for robustness, the timing of T $\alpha 1$ was included in the multivariable logistic model in three different forms, and the findings remain stable. We inferred that the inflammatory response may be stronger in the later stage than in the early stage in COVID-19. Therefore, compared to early use, T $\alpha 1$ use at a later stage may exacerbate the inflammatory response and lead to a poor prognosis. However, data about the inflammatory status at different stages were lacking. More studies are needed to validate our speculation. In addition, we also investigated the impact of T $\alpha 1$ duration on prognosis in COVID-19. In the tertile analysis, we noted that compared to Tertile 1 ( $\leq 3$ days), OR in Tertile 2 was significant while non-significant in Tertile 3 . When we included the duration of T $\alpha 1$ in other two forms, the results became nonsignificant. Therefore, there is a chance that this significant finding may be affected by different cut-off values, and the stability of this result should be verified.

\section{Strengths and Limitations}

To the best of our knowledge, this is the first large, multicenter observational study confirming the association between To1 therapy and increased non-recovery rate in severely ill COVID-19 patients. The main strengths of this study included the large sample size and the use of multiple statistical analyses methods to minimize confounding bias. However, several limitations need to be addressed. First, the use of T $\alpha 1$ for the treatment of COVID-19 patients was mainly at the discretion of clinicians. Thus, the reasons for its use may have been quite varied, such as poor immune status, presence of hepatic disease, severe infection, or simply the clinician's preference. Second, we included most of the covariates, both in a multivariable model and PSM analysis. However, bias caused by potential confounding factors remains possible, such as the time of COVID-19 diagnosis. Third, owing to the retrospective nature of this study, the protocol for T $\alpha 1$ administration was diverse among patients; for instance, the timing of T $\alpha 1$ use was significantly associated with a poor outcome, whereas the duration was not. Thus, rigorously designed randomized controlled studies would be required to reach a more precise conclusion. Fourth, the causal relationship between $\mathrm{T} \alpha 1$ and a poor clinical outcome in the context of COVID-19 cannot be inferred from the current study. Although the results from the PSM supported our hypothesis, our work is observational. Therefore, future prospective studies are needed to verify our results.

\section{CONCLUSIONS}

The hyper-inflammatory response is a hallmark of COVID-19. T $\alpha 1$ use in these patients is significantly associated with an increased non-recovery rate and a poor clinical outcome, especially in individuals with severe disease. As the COVID-19 outbreak is still ongoing, T $\alpha 1$ use in this group of patients should be treated with caution.

\section{DATA AVAILABILITY STATEMENT}

The raw data supporting the conclusions of this article will be made available by the authors, without undue reservation.

\section{ETHICS STATEMENT}

The studies involving human participants were reviewed and approved by the Ethics Committee of the Jin Yin-tan Hospital (KY-2020-03.01). Written informed consent for participation was not required for this study in accordance with the national legislation and the institutional requirements. 


\section{AUTHOR CONTRIBUTIONS}

DC and XD designed the project. YC, LZ, JG, HD, YL,TW, LC, $\mathrm{WL}$, and $\mathrm{WZ}$ carried out the data collection. YS analyzed the data and prepared the figures. JL, YS, ZLW, QX, ZXW, HF and ZL drafted the manuscript. JT and DA provided some writing suggestions. All authors contributed to the article and approved the submitted version.

\section{FUNDING}

This work was supported by the National Natural Science Foundation of China [81873944, 81971869, and 81571943].

\section{REFERENCES}

1. Ruan Q, Yang K, Wang W, Jiang L, Song J. Clinical Predictors of Mortality Due to COVID-19 Based on an Analysis of Data of 150 Patients From Wuhan, China. Intensive Care Med (2020) 46(5):846-8. doi: 10.1007/s00134020-05991-x

2. Huang C, Wang Y, Li X, Ren L, Zhao J, Hu Y, et al. Clinical Features of Patients Infected With 2019 Novel Coronavirus in Wuhan, China. Lancet (2020) 395(10223):497-506. doi: 10.1016/S0140-6736(20)30183-5

3. Moore JB, June CH. Cytokine Release Syndrome in Severe COVID-19. Science (2020) 368(6490):473-4. doi: 10.1126/science.abb8925

4. Yu K, He J, Wu Y, Xie B, Liu X, Wei B, et al. Dysregulated Adaptive Immune Response Contributes to Severe COVID-19. Cell Res (2020) 30(9):814-6. doi: 10.1038/s41422-020-0391-9

5. Subir R, Jagat JM, Kalyan KG. Pros and Cons for Use of Statins in People With Coronavirus Disease-19 (COVID-19). Diabetes Metab Syndr (2020) 14 (5):1225-9. doi: 10.1016/j.dsx.2020.07.011

6. Group RC, Horby P, Lim WS, Emberson JR, Mafham M, Bell JL, et al. Dexamethasone in Hospitalized Patients With Covid-19. N Engl J Med (2021) 384(8):693-704. doi: 10.1056/NEJMoa2021436

7. Liu J, Zhang S, Dong X, Li Z, Xu Q, Feng H, et al. Corticosteroid Treatment in Severe COVID-19 Patients With Acute Respiratory Distress Syndrome. J Clin Invest (2020) 130(12):6417-28. doi: 10.1172/JCI140617

8. Liu Y, Pang Y, Hu Z, Wu M, Wang C, Feng Z, et al. Thymosin Alpha 1 (Talpha1) Reduces the Mortality of Severe COVID-19 by Restoration of Lymphocytopenia and Reversion of Exhausted T Cells. Clin Infect Dis (2020) 71(16):2150-7. doi: 10.1093/cid/ciaa630

9. Sun Q, Xie J, Zheng R, Li X, Chen H, Tong Z, et al. The Effect of Thymosin Alpha1 on Mortality of Critical COVID-19 Patients: A Multicenter Retrospective Study. Int Immunopharmacol (2021) 90:107143. doi: 10.1016/ j.intimp.2020.107143

10. Wang Y, Yan X, Huang C, Sun Y, Yao C, Lin Y, et al. Risk Factors of Mortality and Contribution of Treatment in Patients Infected With COVID-19: A Retrospective Propensity Score Matched Study. Curr Med Res Opin (2021) 37 (1):13-9. doi: 10.1080/03007995.2020.1853508

11. Wu M, Ji JJ, Zhong L, Shao ZY, Xie QF, Liu ZY, et al. Thymosin Alpha1 Therapy in Critically Ill Patients With COVID-19: A Multicenter Retrospective Cohort Study. Int Immunopharmacol (2020) 88:106873. doi: 10.1016/j.intimp.2020.106873

12. Romani L, Bistoni F, Montagnoli C, Gaziano R, Bozza S, Bonifazi P, et al. Thymosin Alpha1: An Endogenous Regulator of Inflammation, Immunity, and Tolerance. Ann NY Acad Sci (2007) 1112:326-38. doi: 10.1196/annals.1415.002

13. Pei F, Guan X, Wu J. Thymosin Alpha 1 Treatment for Patients With Sepsis. Expert Opin Biol Ther (2018) 18(sup1):71-6. doi: 10.1080/14712598.2018.1484104

14. Naylor PH, Mutchnick MG. Immunotherapy for Hepatitis B in the Direct Acting Antiviral Era: Reevaluating the Thymosin Alphal Efficacy Trials in the Light of a Combination Therapy Approach. J Viral Hepat (2018) 25(1):4-9. doi: $10.1111 /$ jvh. 12807

\section{ACKNOWLEDGMENTS}

We would like to thank all the staff at the five selected hospitals, who were involved in COVID-19 patient care, as well as all the patients who took part in the study and their families.

\section{SUPPLEMENTARY MATERIAL}

The Supplementary Material for this article can be found online at: https://www.frontiersin.org/articles/10.3389/fimmu.2021. 673693/full\#supplementary-material

15. Romani L, Oikonomou V, Moretti S, Iannitti RG, D’Adamo MC, Villella VR, et al. Thymosin Alpha1 Represents a Potential Potent Single-Molecule-Based Therapy for Cystic Fibrosis. Nat Med (2017) 23(5):590-600. doi: 10.1038/ nm.4305

16. Wu J, Zhou L, Liu J, Ma G, Kou Q, He Z, et al. The Efficacy of Thymosin Alpha 1 for Severe Sepsis (ETASS): A Multicenter, Single-Blind, Randomized and Controlled Trial. Crit Care (2013) 17(1):R8. doi: 10.1186/cc11932

17. Soy M, Keser G, Atagunduz P, Tabak F, Atagunduz I, Kayhan S. Cytokine Storm in COVID-19: Pathogenesis and Overview of Anti-Inflammatory Agents Used in Treatment. Clin Rheumatol (2020) 39(7):2085-94. doi: 10.1007/s10067-020-05190-5

18. Soldati G, Giannasi G, Smargiassi A, Inchingolo R, Demi L. ContrastEnhanced Ultrasound in Patients With COVID-19: Pneumonia, Acute Respiratory Distress Syndrome, or Something Else? J Ultrasound Med (2020) 39(12):2483-9. doi: 10.1002/jum.15338

19. National Health Commission of the People's Republic of China. Diagnosis and Treatment of COVID-19 Guidelines (Trial Version 7). Available at: http:// www.nhc.gov.cn/xcs/zhengcwj/202003/46c9294a7dfe4cef80dc7f5912eb1989/ files/ce3e6945832a438eaae415350a8ce964.pdf.

20. Vincent JL, Moreno R, Takala J, Willatts S, De Mendonca A, Bruining H, et al. The SOFA (Sepsis-Related Organ Failure Assessment) Score to Describe Organ Dysfunction/Failure. On Behalf of the Working Group on SepsisRelated Problems of the European Society of Intensive Care Medicine. Intensive Care Med (1996) 22(7):707-10. doi: 10.1007/BF01709751

21. Knaus WA, Draper EA, Wagner DP, Zimmerman JE. APACHE II: A Severity of Disease Classification System. Crit Care Med (1985) 13(10):818-29. doi: 10.1097/00003246-198510000-00009

22. Force ADT, Ranieri VM, Rubenfeld GD, Thompson BT, Ferguson ND, Caldwell E, et al. Acute Respiratory Distress Syndrome: The Berlin Definition. JAMA (2012) 307(23):2526-33. doi: 10.1001/jama.2012.5669

23. Khwaja A. KDIGO Clinical Practice Guidelines for Acute Kidney Injury. Nephron Clin Pract (2012) 120(4):c179-84. doi: 10.1159/000339789

24. Huang I, Pranata R. Lymphopenia in Severe Coronavirus Disease-2019 (COVID-19): Systematic Review and Meta-Analysis. J Intensive Care (2020) 8:36. doi: 10.1186/s40560-020-00453-4

25. Yu Y, Xu D, Fu S, Zhang J, Yang X, Xu L, et al. Patients With COVID-19 in 19 ICUs in Wuhan, China: A Cross-Sectional Study. Crit Care (2020) 24(1):219. doi: 10.1186/s13054-020-02939-x

26. Zhang L, Liu Y. Potential Interventions for Novel Coronavirus in China: A Systematic Review. J Med Virol (2020) 92(5):479-90. doi: 10.1002/jmv.25707

27. Shao C, Tian G, Huang Y, Liang W, Zheng H, Wei J, et al. Thymosin Alpha-1Transformed Bifidobacterium Promotes T Cell Proliferation and Maturation in Mice by Oral Administration. Int Immunopharmacol (2013) 15(3):646-53. doi: 10.1016/j.intimp.2012.12.031

28. Serafino A, Pierimarchi P, Pica F, Andreola F, Gaziano R, Moroni N, et al. Thymosin Alphal as a Stimulatory Agent of Innate Cell-Mediated Immune Response. Ann NY Acad Sci (2012) 1270:13-20. doi: 10.1111/j.17496632.2012.06707.x 
29. Matteucci C, Minutolo A, Balestrieri E, Petrone V, Fanelli M, Malagnino V, et al. Thymosin Alpha 1 Mitigates Cytokine Storm in Blood Cells From Coronavirus Disease 2019 Patients. Open Forum Infect Dis (2021) 8(1): ofaa588. doi: 10.1093/ofid/ofaa588

30. Giacomini E, Severa M, Cruciani M, Etna MP, Rizzo F, Pardini M, et al. Dual Effect of Thymosin Alpha 1 on Human Monocyte-Derived Dendritic Cell In Vitro Stimulated With Viral and Bacterial Toll-Like Receptor Agonists. Expert Opin Biol Ther (2015) 15 Suppl 1:S59-70. doi: 10.1517/ 14712598.2015.1019460

31. Shi Y, Wang Y, Shao C, Huang J, Gan J, Huang X, et al. COVID-19 Infection: The Perspectives on Immune Responses. Cell Death Differ (2020) 27(5):14514. doi: 10.1038/s41418-020-0530-3

32. Wang D, Hu B, Hu C, Zhu F, Liu X, Zhang J, et al. Clinical Characteristics of 138 Hospitalized Patients With 2019 Novel Coronavirus-Infected Pneumonia in Wuhan, China. JAMA (2020) 323(11):1061-9. doi: 10.1001/ jama.2020.1585

33. King R, Tuthill C. Immune Modulation With Thymosin Alpha 1 Treatment. Vitam Horm (2016) 102:151-78. doi: 10.1016/bs.vh.2016.04.003
Conflict of Interest: The authors declare that the research was conducted in the absence of any commercial or financial relationships that could be construed as a potential conflict of interest.

Publisher's Note: All claims expressed in this article are solely those of the authors and do not necessarily represent those of their affiliated organizations, or those of the publisher, the editors and the reviewers. Any product that may be evaluated in this article, or claim that may be made by its manufacturer, is not guaranteed or endorsed by the publisher.

Copyright (C) 2021 Liu, Shen, Wen, Xu, Wu, Feng, Li, Dong, Huang, Guo, Zhang, Chen, Li, Zhu, Du, Liu, Wang, Chen, Teboul, Annane and Chen. This is an openaccess article distributed under the terms of the Creative Commons Attribution License (CC BY). The use, distribution or reproduction in other forums is permitted, provided the original author(s) and the copyright owner(s) are credited and that the original publication in this journal is cited, in accordance with accepted academic practice. No use, distribution or reproduction is permitted which does not comply with these terms. 